\title{
Naringin and bone marrow mesenchymal stem cells repair articular cartilage defects in rabbit knees through the transforming growth factor- $\beta$ superfamily signaling pathway
}

\author{
CHAO YE $^{1^{*}}$, JING CHEN $^{2 *}$, YI QU ${ }^{1}$, HANG LIU $^{3}$, JUNXING YAN $^{4}$, YINGDONG LU $^{5}$, \\ ZHENG YANG $^{6}$, FENGXIAN WANG ${ }^{1}$ and PENGYANG LI ${ }^{1}$
}

\begin{abstract}
${ }^{1}$ Orthopedics Department, Dongzhimen Hospital, Beijing University of Chinese Medicine, Beijing 100700;
${ }^{2}$ Preventative Treatment of Disease Department, The Third Affiliated Hospital, Beijing University of Chinese Medicine, Beijing 100029; ${ }^{3}$ Orthopedics Department, Huguosi Hospital, Beijing University of Chinese Medicine, Beijing 100035; ${ }^{4}$ Orthopedics Department, Tongzhou District Hospital of Integrated Traditional Chinese Medicine and Western Medicine,

Beijing 101100; ${ }^{5}$ Pathology Department, Guang'anmen Hospital, China Academy of Chinese Medical Sciences, Beijing 100053; ${ }^{6}$ SATCM Key Laboratory of Renowned Physician and Classical Formula,

Beijing University of Chinese Medicine, Beijing 100029, P.R. China
\end{abstract}

Received July 25, 2019; Accepted April 21, 2020

DOI: $10.3892 / \mathrm{etm} .2020 .9187$

\begin{abstract}
The present study aimed to assess the effect of a combination of naringin and rabbit bone marrow mesenchymal stem cells (BMSCs) on the repair of cartilage defects in rabbit knee joints and to assess possible involvement of the transforming growth factor- $\beta$ (TGF- $\beta$ ) signaling pathway in this process. After establishing an articular cartilage defect model in rabbit knees, 20 New Zealand rabbits were divided into a sham operation group (Sham), a model group (Mod), a naringin treatment group (Nar), a BMSC group (BMSCs) and a naringin + BMSC group (Nar/BMSCs). At 12 weeks after treatment, the cartilage was evaluated using the International Cartilage Repair Society (ICRS)'s macroscopic evaluation of cartilage repair scale, the ICRS's visual histological assessment scale, the Modified O'Driscoll grading system, histological staining (hematoxylin and eosin staining, toluidine blue staining and safranin $\mathrm{O}$ staining) and immunohistochemical staining (type-II collagen, TGF- $\beta 3$ and SOX-9 immunostaining). Using the above grading systems to quantify the extent of repair, histological quantification and macro quantification of joint tissue repair showed that the Nar/BMSCs group displayed
\end{abstract}

Correspondence to: Dr Pengyang Li, Orthopedics Department, Dongzhimen Hospital, Beijing University of Chinese Medicine, 5 Haiyuncang Street, Beijing 100700, P.R. China

E-mail: lipengyangbucm@163.com

*Contributed equally

Key words: naringin, rabbit, knee joint, cartilage defect, bone mesenchymal stem cells, transforming growth factor- $\beta$ superfamily signaling pathway repair after treatment in comparison to the untreated Mod group. Among the injury model groups (Mod, Nar, BMSCs and Nar/BMSCs), the Nar/BMSCs group displayed the highest degree of morphological repair. The results of histological and immunohistochemical staining of the repaired region of the joint defect indicated that the BMSCs had a satisfactory effect on the repair of the joint structure but had a poor effect on the repair of cartilage quality. The Nar/BMSCs group displayed satisfactory therapeutic effects on both repair of the joint structure and cartilage quality. The expression level of type-II collagen was high in the Nar/BMSCs group. Additionally, staining of TGF- $\beta 3$ and SOX-9 in the Nar/BMSCs group was the strongest compared with that of any other group in the present study. Naringin and/BMSCs together demonstrated a more efficient repair effect on articular cartilage defects in rabbit knees than the use of either treatment alone in terms of joint structure and cartilage quality. One potential mechanism of naringin action may be through activation and continuous regulation of the TGF- $\beta$ superfamily signaling pathway, which can promote BMSCs to differentiate into chondrocytes.

\section{Introduction}

Knee osteoarthritis is a degenerative joint disease induced by articular cartilage degeneration. Its main clinical features are arthralgia and joint deformity and dysfunction (1). It is characterized by a high incidence and recurrent seizures of the joint and worsens with time. As the condition advances, prosthetic replacement surgery is usually required. Over a million people worldwide receive new diagnoses of articular cartilage disease annually (2) and the condition lowers the quality of life of these patients (3).

Articular cartilage, an avascular tissue, can be classified into hyaline cartilage, fibrocartilage and elastic cartilage. Hyaline cartilage is located on the surface of the knee joint 
and has critical functions that differ from the other two types of cartilage, such as the secretion of lubricating substances and friction reduction. Hyaline cartilage plays a vital role in maintaining the normal functions of the knee joint (4).

It is well accepted that knee joint cartilage has a poor blood supply. Moreover, it lacks stromal stem cells and has an extremely poor ability for self-repair (5). When cartilage is repaired, it is usually spontaneously repaired into fibrocartilage, not hyaline cartilage, therefore losing the extra function of this cartilage form (6). There are a number of differences between fibrocartilage and hyaline cartilage in terms of tissue structure and mechanical properties (7). Articular cartilage defects, the predominant factor in the pathological process of osteoarthritis, lead to degeneration of the joint (8).

Much attention has been paid to natural small molecule compounds based on plant derivatives for the potential treatment of osteoarticular disease, attributed to the feasibility of their collection and their bioactivity and therapeutic effects (9). A previous study indicated that these compounds may be beneficial in the treatment of osteoarticular diseases through the regulation of signaling transduction pathways (10).

In traditional Chinese medicine (TCM), the pathogenesis of osteoarthritis is thought to involve liver and kidney deficiencies, as well as malnutrition of the muscles and bones (11-13). Drynaria fortune i is a commonly used treatment in TCM which is thought to benefit the liver and kidneys, strengthen tendons and bones and target the key treatment points of osteoarthritis. Drynaria fortune $i$ is thought to be therapeutically beneficial in the treatment of knee osteoarthritis (14). The present study was designed to investigate the specific mechanism of action of Drynaria fortunei, as current knowledge is lacking. Naringin is a plant derivative and also an active ingredient of Drynaria fortunei and is thought to have the same effect as the corresponding dosage of the crude Drynaria fortune (15).

Mesenchymal stem cells (MSCs) are cells with marked proliferative abilities and the ability to differentiate into cartilage cells (16). Myeloid tissue is one of the main sources of MSCs. MSCs that exist in the bone marrow are termed bone marrow mesenchymal stem cells (BMSCs). They are one of the most commonly used cells in research into articular cartilage repair (17). A number of compounds are thought to exhibit potential for promotion of the differentiation of BMSCs, such as insulin-like growth factor I and vascular endothelial growth factor (18).

The transforming growth factor (TGF)- $\beta$ superfamily includes TGF- $\beta$ s and bone morphogenetic proteins. The TGF- $\beta$ signaling pathway plays a vital role in cartilage cell proliferation and differentiation. SOX-9, a downstream factor in this pathway, plays an indispensable function in articular cartilage differentiation and is a transcriptional regulator of chondrogenesis (19). SOX-9 is necessary to support a normal cartilage cell phenotype (20). Overexpression of SOX-9 results in increased synthesis of aggrecan, which can provide a suitable living environment for articular cartilage (21).

Naringin, the active ingredient of Drynaria fortunei, can be taken orally or injected directly into a cartilage defect site in combination with BMSCs and has previously been suggested to stimulate the repair of articular cartilage defects in rabbit knees (22). The present study aimed to conduct an in-depth investigation into the repair if joint structure and cartilage and assess the role of the TGF- $\beta$ superfamily signaling pathway in this process. A scoring system, histological staining and immunohistochemical staining were used to quantify the efficacy of naringin induced repair and the potential mechanisms of naringin action were explored (Fig. 1).

\section{Materials and methods}

Extraction and separation of rabbit BMSCs. Following a previously published protocol (23), a 2-week-old New Zealand rabbit was used for the collection of BMSCs. Rabbits were anesthetized by intravenous injection of $30 \mathrm{mg} / \mathrm{kg}$ pentobarbital sodium into the ear margin. A bone marrow puncture needle was then inserted into the posterior superior iliac spine of the rabbit. A total of $20 \mathrm{ml}$ of low molecular weight heparin calcium (Tianjin Chase Sun Pharmaceutical Co., Ltd.) was injected to extract the bone marrow. The inner wall of the syringe was moistened with low molecular weight heparin 5 min before extraction. The extracted bone marrow was then injected into a sterile centrifuge tube and was diluted 1:5 with L-DMEM (Beijing Solarbio Science \& Technology Co., Ltd.). The bone marrow-DMEM mixture was centrifuged at $1,200 \mathrm{x} \mathrm{g}$ for $5 \mathrm{~min}$ at $25^{\circ} \mathrm{C}$. The supernatant was discarded and subsequently cells were resuspended in $20 \mathrm{ml}$ DMEM containing 10\% fetal calf serum (Gibco; Thermo Fisher Scientific, Inc.). The extracted cells were added to a culture flask and placed in an incubator at $37^{\circ} \mathrm{C}$ and $5 \% \mathrm{CO}_{2}$. The medium was replaced with fresh medium $48 \mathrm{~h}$ later. The full-marrow adherent method (24) was employed for separation and purification of rabbit BMSCs. The culture medium was replaced every 3 days until the cells reached $80 \%$ confluency, when they were passaged for further experiments.

Preparation of tested animals. In total, 20 New Zealand rabbits (3-month-old, male) were selected, with a weight of $\sim 2.5 \mathrm{~kg}$ prior to surgery. They were placed in separate cages and allowed to freely move within the cage. The animals had access to food and water ad libitum. A 12-h light-dark cycle was maintained. The temperature was maintained at $21-25^{\circ} \mathrm{C}$ and the relative humidity was maintained at $40-70 \%$.

The animals were acclimatized for $\geq 7$ days before subsequent experiments. The present study followed the National Institutes of Health Guidelines Regarding the Care and Use of Laboratory Animals. Animal treatment in the experimental process conformed to the relevant regulations on ethical standards of animals specified in the Guiding Proposal for Being Kind to Experimental Animals issued by the Ministry of Science and Technology and in Ethical Issues in Animal Experimentation $(11,25)$. The animal experiments were approved by the Science and Technology Department of Beijing University of Chinese Medicine (Beijing, China) and the animal ethics review committee of the Institute of Basic Theories of Chinese medicine, Chinese Academy of Chinese Medical Sciences, approval no. 201706058 (Beijing, China).

Animal models. Animal models for articular cartilage defects were prepared according to a previously described method $(22,23)$. New Zealand rabbits were purchased from Beijing Changyang Xishan Experimental Animal Farm. Rabbits were anesthetized using intravenous administration 
Table I. New Zealand white rabbit baseline information.

\begin{tabular}{lccccc}
\hline Parameter & Sham operation group & Model group & Naringin group & BMSCs group & Naringin+BMSCs group \\
\hline Weight, kg & $2.80 \pm 0.41$ & $2.78 \pm 0.17$ & $2.83 \pm 0.22$ & $3.10 \pm 0.24$ & $3.20 \pm 0.34$ \\
Sex, male/female & $4 / 0$ & $4 / 0$ & $4 / 0$ & $4 / 0$ & $4 / 0$ \\
Age, month & 6 & 6 & 6 & 6 & 6 \\
\hline
\end{tabular}

of $3 \%$ sodium pentobarbital $(30 \mathrm{mg} / \mathrm{kg})$ in the ear vein. Knee tissues were cut layer by layer under aseptic conditions after anesthesia (skin, subcutaneous tissue, muscle, joint capsule). A corneal trephine (Beijing North Sanyou Medical Devices Co., Ltd.) was used to drill a hole on the articular surface of the trochlea from the femoral condyle to the subchondral bone, with a diameter of $5 \mathrm{~mm}$ and a depth of $3 \mathrm{~mm}$. The defect site was filled according to the experimental scheme. The patella was reset after completion. The knee joint capsule and skin were sutured. Penicillin $\left(10 \times 10^{4} \mathrm{U}\right)$ was injected intramuscularly postoperatively each day for 3 days. All rabbits were allowed to move their knee joints freely in their cages without restriction and clinical signs were observed daily. No animal was excluded owing to abnormal clinical findings. The rabbits were anesthetized using intravenous administration of $3 \%$ sodium pentobarbital $(30 \mathrm{mg} / \mathrm{kg})$ in the ear vein. Measurement of anesthetic depth, respiratory rate and pattern and assessment of mucous membrane color and heart rate monitoring was performed throughout anesthesia. The animals were sacrificed by an intravenous air bolus injection into the ear vein containing $30 \mathrm{ml}$ air, which was injected into the ear margin. Animal death was confirmed by respiratory arrest, cardiac arrest and changes in mucosal color. Rabbits in different groups were sacrificed at the 12th week after surgery. There were no statistically significant differences in body weight among the different groups (Table I).

Preparation of naringin solution. A naringin solution was prepared with $10 \mathrm{~g}$ naringin and $100 \mathrm{ml}$ deionized water. The gavage concentration (calculated according to the weight of naringin) was $0.0084 \mathrm{~g} / \mathrm{kg}$ daily $(26,27)$.

Laboratory animal grouping and intervention method. All 20 New Zealand rabbits were randomly distributed into 5 groups (4 rabbits and 8 knee joints in each group). In the sham operation group (sham), the joint capsule was cut and seamed, while the knee joint was not treated. In the model group (Mod), deionized water was administered via gavage every day after successful establishment of the articular cartilage defect model. In the naringin group (Nar), naringin solution was administered via gavage every day after successful establishment of the articular cartilage defect model. In the BMSCs group (BMSCs), rabbit BMSCs were immediately implanted at the joint defect site after successful establishment of the articular cartilage defect model. Moreover, deionized water was administered via gavage every day. In the naringin + BMSCs group (Nar/BMSCs), after successful establishment of the articular cartilage defect model, BMSCs were implanted at the defect site immediately and the naringin solution was administered to the rabbits every day. The daily volume of
Table II. International Cartilage Repair Society macroscopic evaluation of cartilage repair.

Category Score

Degree of defect repair

In level with surrounding cartilage 4

$75 \%$ repair of defect depth 4

$50 \%$ repair of defect depth

$25 \%$ repair of defect depth

No repair of defect depth

Integration to border zone

Complete integration with surrounding cartilage 4

Demarcating border-1 $\mathrm{mm}$

Three-quarters of graft integrated, one-quarter with

a notable border- $1 \mathrm{~mm}$ in width

One-half of graft integrated with surrounding cartilage, 1

one-half with a notable border-1 $\mathrm{mm}$

From no contact, to one-quarter of graft integrated

with surrounding cartilage

Macroscopic appearance

Intact smooth surface

4

Fibrillated surface

3

Small, scattered fissures or cracks $\quad 2$

Several small or few large fissures $\quad 1$

Total degeneration of grafted area $\quad 0$

Overall repair assessment

Grade I, normal

Grade II, nearly normal $\quad$ 8-11

Grade III, abnormal 4-7

Grade IV, severely abnormal $\quad 1-3$

deionized water in the Mod and BMSC groups was $0.25 \mathrm{ml}$. The volume of gavage in Nar and Nar/BMSCs groups was $0.25 \mathrm{ml}$.

Macroevaluation. In order to reexamine the intra-articular structure and to cut out the complete double knee joint of rabbits, arthrotomy was carried out using the same approach as that used during modeling. The International Cartilage Repair Society (ICRS) macroscopic evaluation of cartilage repair (Table II) was adopted to systematically evaluate the degree of articular cartilage repair in the different groups (28). The main parameters monitored were the degree of defect repair, integration to the border zone and macroscopic appearance. All specimens were independently evaluated by two professionals 
with intermediate professional titles. The investigators who performed the evaluations were blind to the treatment.

Histological and immunohistochemical evaluation. The specimens were fixed in $10 \%$ neutral formalin at room temperature for $24 \mathrm{~h}$. For decalcification the specimens were placed in EDTA-buffered solution (Beijing Zhongshan Jinqiao Biotechnology Co. Ltd.) for 4 weeks. The decalcifying solution was changed twice per week. The articular cartilage of the repaired area was cut out after obtaining satisfactory decalcification (Achieved when the syringe needle punctured the tissue without resistance and the tissue was soft and elastic.), so as to be trimmed into a sheet with a thickness of $4 \mathrm{~mm}$. Freshly prepared $4 \%$ methanal was used for fixation for $48 \mathrm{~h}$ at room temperature, followed by paraffin embedding and slicing. The specimens were cut into $4-\mu \mathrm{m}$ thick slices for histological and immunohistochemical staining, including hematoxylin and eosin (H\&E) staining (H\&E staining kit; Beijing Solarbio Science \& Technology Co., Ltd.) to observe morphological changes in cartilage cells; toluidine blue staining (Toluidine Blue O cartilage stain solution; Beijing Solarbio Science \& Technology Co., Ltd.) to observe the secretion of acid mucopolysaccharide and indirectly evaluate the cartilage matrix; safranin O staining (Safranine O-Fast Green FCF cartilage stain kit; Beijing Solarbio Science \& Technology Co., Ltd.) to display the conditions of osteogenesis and chondrogenesis; type-II collagen immunostaining (rabbit anti-collagen II antibody; Beijing Bioss Biotechnology Co., Ltd.) to detect specific indicators of cartilage cells and exhibit the content and quality of hyaline cartilage. Slices were subsequently blocked in $3 \% \mathrm{H}_{2} \mathrm{O}_{2}$ (Beijing Zhongshan Jinqiao Biotechnology Co. Ltd.) for $10 \mathrm{~min}$ at room temperature before staining. Slices were incubated with rabbit anti-collagen II antibody (Beijing Bioss Biotechnology Co., Ltd.; cat. no. bs-10589R; 1:200) at $37^{\circ} \mathrm{C}$ for $2 \mathrm{~h}$, followed by incubation with horseradish peroxidase conjugated goat anti-rabbit/mouse secondary antibody (1:1; cat. no. PV6000; Beijing Zhongshan Jinqiao Biotechnology Co., Ltd) at room temperature for $30 \mathrm{~min}$. TGF- $\beta 3 \mathrm{immu}-$ nostaining was performed to evaluate the expression and secretion of TGF- $\beta 3$. Slices were incubated with rabbit anti-TGF- $\beta 3$ antibody (Rabbit anti-TGF- $\beta 3$ antibody; Beijing Bioss Biotechnology Co., Ltd.; cat. no. bs-1910R; 1:300) at $37^{\circ} \mathrm{C}$ for $2 \mathrm{~h}$, followed by incubation with horseradish peroxidase conjugated goat anti-rabbit secondary antibody (1:1; cat. no. PV6000; Beijing Zhongshan Jinqiao Biotechnology Co., Ltd) at room temperature for $30 \mathrm{~min}$. SOX-9 immunostaining was used to evaluate the expression and secretion of SOX-9. Slices were incubated rabbit anti-SOX9 antibody (rabbit anti-SOX9 antibody; Beijing Bioss Biotechnology Co., Ltd.; cat. no. bs-10725R; $1: 200$ ) at $37^{\circ} \mathrm{C}$ for $2 \mathrm{~h}$, followed by incubation with horseradish peroxidase conjugated goat anti-rabbit secondary antibody (1:1; PV6000; Beijing Zhongshan Jinqiao Biotechnology Co., Ltd.) at room temperature for $20 \mathrm{~min}$. After staining, the slices were photographed and examined using an optical microscope (Nikon Eclipse 600; Nikon Corporation) equipped with a digital camera (Nikon DXM1200F; Nikon Corporation) to record images of the stained slices.

A total of 40 slices were prepared from each rabbit and from each slice three images were captured. All staining procedures were conducted according to the manufacturers' instructions.
Table III. International Cartilage Repair Society visual histological assessment scale.

\begin{tabular}{lc}
\hline Feature & Sco \\
\hline Surface & 3 \\
Smooth/continuous & 0 \\
Discontinuities/irregularities & 3 \\
Matrix & 2 \\
Hyaline & 1 \\
Mixture, hyaline/fibrocartilage & 0 \\
Fibrocartilage & \\
Fibrous tissue & 3 \\
Cell distribution & 2 \\
Columnar & 1 \\
Mixed/columnar-clusters & 0 \\
Clusters & \\
Individual cells/disorganized & 2 \\
Cell population viability & 1 \\
Predominantly viable & 0 \\
Partially viable &
\end{tabular}

Subchondral bone

Normal 3

Increased remodeling

Bone necrosis/granulation tissue

Detached/fracture/callus at base 0

Cartilage mineralization salcified cartilage)

Normal

Abnormal/inappropriate location

Toluidine blue stain

Normal

4

Slight reduction

3

Moderate reduction $\quad 2$

Severe reduction $\quad 1$

No staining

Percent toluidine blue in defect

$75-100 \%$

$50-75 \%$

3

$25-50 \%$

2

$0-25 \%$

No toluidine blue staining

The ICRS Visual Histological Assessment Scale (Table III) and the modified O'Driscoll grading system (Table IV) were utilized to evaluate articular cartilage repair level in the different groups (29-31). The main parameters included surface, matrix and cell distribution, cell population viability, nature of the predominant tissue, structural characteristics and absence of cellular changes due to degeneration. All specimens were independently evaluated by two professionals.

Statistical analysis. SPSS 19.0 software (IBM Corp.) and Prism 7 (GraphPad Software, Inc.) were used for statistical analysis. Data are presented as the mean value \pm SD. All 
Table IV. Modified O'Driscoll grading system.

A, Nature of the predominant tissue

\begin{tabular}{lc}
\hline Parameter & Score \\
\hline Cellular morphology & \\
Hyaline articular cartilage & 4 \\
Incompletely differentiated mesenchyme & 2 \\
Fibrous tissue or bone & 0 \\
Safranin-O staining of the matrix & \\
Normal or nearly normal & 3 \\
Moderate & 2 \\
Slight & 1 \\
None & 0
\end{tabular}

B, Structural characteristics

Parameter

Score

Surface regularity

Smooth and intact

Superficial horizontal lamination

Fissures, $25-100 \%$ of the thickness

Severe disruption. including fibrillation

Structural integrity

Normal

Slight disruption. including cysts

Severe disintegration

Thickness

100 per cent of normal adjacent cartilage

$50-100$ per cent of normal cartilage

$0-50$ per cent of normal cartilage

Bonding to the adjacent cartilage

Bonded at both ends of graft

Bonded at one end, or partially at both ends

Not bonded

C, Freedom from cellular changes of degeneration

Parameter

Score

Hypocellularity

Normal cellularity

Slight hypocellularity

Moderate hypocellularity

Severe hypocellularity

Chondrocyte clustering

No clusters

$<25$ per cent of the cells

25-100 per cent of the cells

Freedom from degenerative changes in adjacent cartilage

Normal cellularity. no clusters, normal staining

Normal cellularity, mild clusters, moderate staining

Mild or moderate hypocellularity, slight staining

Severe hypocellularity. poor or no staining statistical inferences were subjected to two-sided tests. $\mathrm{P}<0.05$ was considered to indicate a statistically significant difference. The confidence interval of parameter estimation was $95 \%$. Statistical differences were analyzed using Kruskal-Wallis tests followed by post-hoc testing using Dunn's multiple comparison tests.

\section{Results}

Morphological observation of tissue repair tissue on the joint defect site. The effect of each treatment on the repair of the surface structure of the articular cartilage defects was studied on the basis of morphological observations. 'Degree of defect repair', 'In level with the surrounding cartilage', 'Integration to border zone', 'Macroscopic appearance' markers.

The main findings were as follows: i) Sham group: There were no surface defects on the knee joint; however, there was a slight abrasion at the edge (Fig. 2A). ii) Mod group: The defect site displayed some tissue granulation with an obvious sunken defect area. There were obvious differences between the areas of normal cartilage tissue and the areas of regenerated tissue. Moreover, an obvious boundary existed between the areas of normal tissue and the areas of defective tissue (Fig. 2B). iii) Naringin group: At the defect site, tissue was semi-transparent and granulated. The defect was not completely filled with regenerated tissue. Compared with the Mod group, the defect area was smaller, with a reduced depth. The boundary between the defect and the surrounding cartilage tissue was fuzzy but distinguishable (Fig. 2C). iv) BMSCs group: Tissue at the defect appeared transparent, in a similar manner to the cartilage tissue. When compared with the Mod group, the defect area was smaller, the depth was shallow, having clearly been reduced and the boundary appeared fuzzy. Although the thickness of the regenerated cartilage was similar to that of the surrounding normal cartilage, the area at the edge of the defect was not as smooth as that observed in normal cartilage (Fig. 2D). v) Nar/BMSCs group: The repaired defect site was integrated with the surrounding tissue. Repairing tissue at the defect site had become milk white, semi-transparent and smooth. It was hard to distinguish repaired tissue at the defect site from the surrounding normal cartilage tissue. The defect site had also developed a smooth surface (Fig. 2E). The characteristic manifestations of the above groups can also be found in the corresponding images before the index detection (Fig. 2F-J). In summary morphological observations in the Naringin group and in the BMSCs group suggested better tissue healing than in the Mod group. However, the Nar/BMSCs group showed the most signs of healing from the defect model animals (Fig. 2).

Pathological staining and observation of the location of repaired tissues at the joint defect site. The repair of joint tissues was observed. The structural repairs to the articular cartilage defects in the treatment groups administered BMSCs were semi-transparent and there were no clear signs of inflammation, however, in the groups that were not administered BMSCs, articular cartilage defects were not smooth and the boundaries between new tissue and surrounding undamaged tissues were readily observed. The treatment groups administered BMSCs appeared to display improved structural repair 


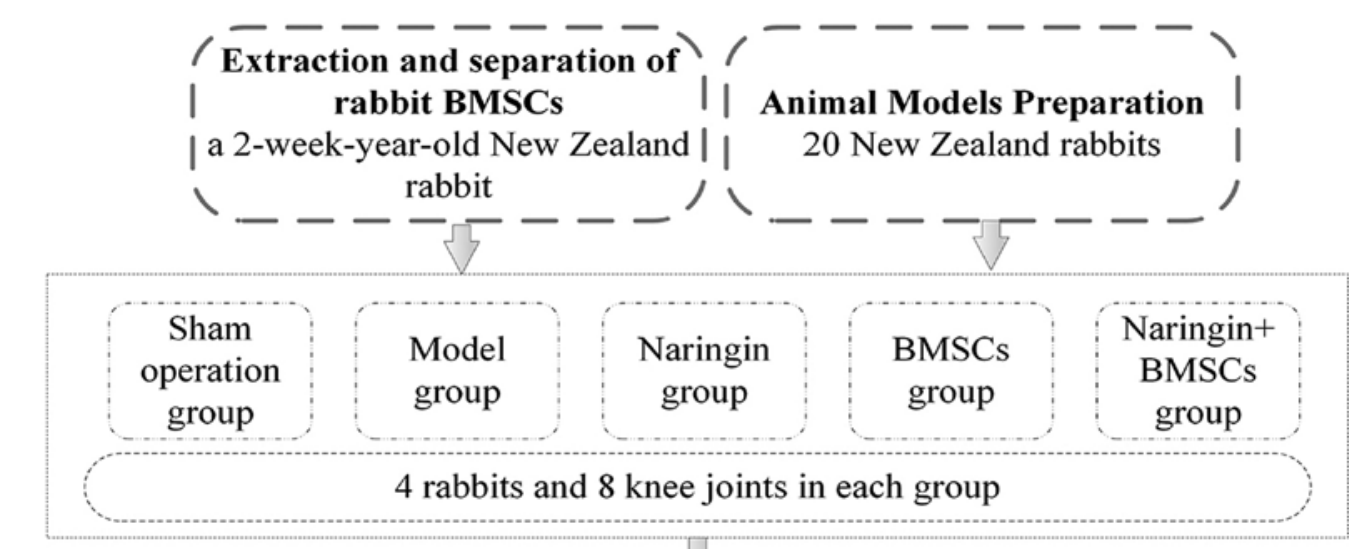

Arthrotomy was conducted on the same admission passage at the time of modeling so as to re-check structure in the joint and to cut out complete double knee joint of rabbit

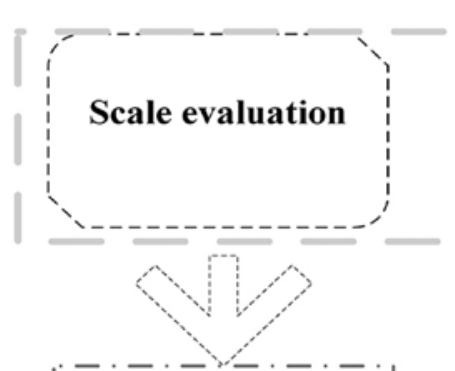

!(1) $\mathrm{i} \overline{\mathrm{CR}} \overline{\mathrm{S}}$ I macroscopic i evaluation of i cartilage repair scale i (2)ICRS Visual Histological ! Assessment Scale ( (3)modified I O'Driscoll grading i. system
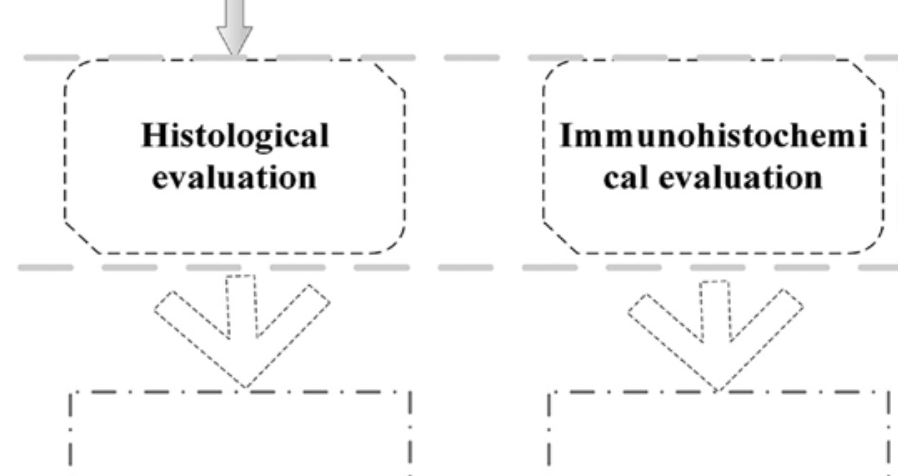

i (1)Type-II collagen immunostaining (2)TGF- $\beta 3$ immunostaining ! (3)SOX-9 I immunostaining । i. $-\cdot-\cdot-\cdot-\cdot-i$

An effective ingredient of the TCM drug Drynaria fortunei, and BMSCs were able to stimulate the repair of articular cartilage defects in rabbit knees. The combined application of these treatments on the joint structure repair and articular cartilage repair quality delivered improved results.

Figure 1. Flow chart describing the structure of experiments performed to evaluate naringin and bone marrow mesenchymal stem cell induced repair of articular cartilage defects in rabbit knees. BMSCs, bone mesenchymal stem cells; ICRS, International Cartilage Repair Society; TGF-3, transforming growth factor- $\beta 3$; SOX-9, sry-type high-mobility-group box-9.

to those that were not administered BMSCs. In terms of the quality of the repair of articular cartilage defects, in the groups that were not administered naringin, cartilage damage was obvious, the hyaline cartilage was severely worn, the thickness of the cartilage layer was reduced and the tide mark was blurred. However, in the groups administered naringin, hyaline cartilage appeared at the site of the cartilage damage, and the tide line was clear, which was close to the nature of surrounding tissue. The Nar/BMSCs group was observed to have the most efficient repair regarding structural and quality repairs of the articular cartilage defects, as shown in Fig. 3.
H\&E staining was used to observe the cartilage morphology. Cells in the repaired tissues of the Mod group were arranged irregularly. Moreover, an obvious gap could be observed (Fig. 3B). The Nar group was observed to have poor structural repair at the defect site. Although the number of repairing cells was limited, the structures and arrangements of the repairing cells generated at the defect site were similar to those of the surrounding normal cartilage cells (Fig. 3C). The BMSCs group was observed to have an efficient overall contour restoration; however, the edge of the repairing tissue was irregular, with obvious gaps (Fig. 3D). The regenerative 


\section{External image of the knee joint in rabbits \\ (Immediately after} taking materials)
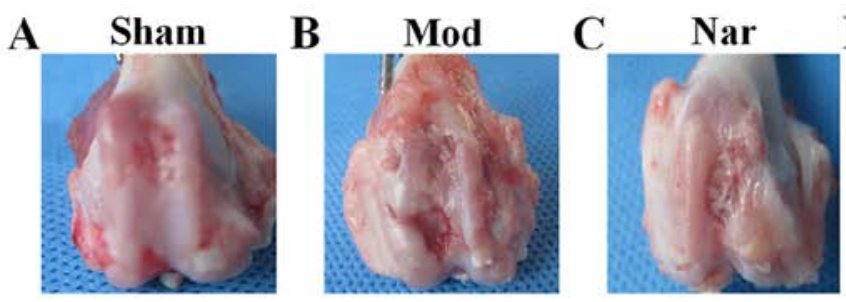

D
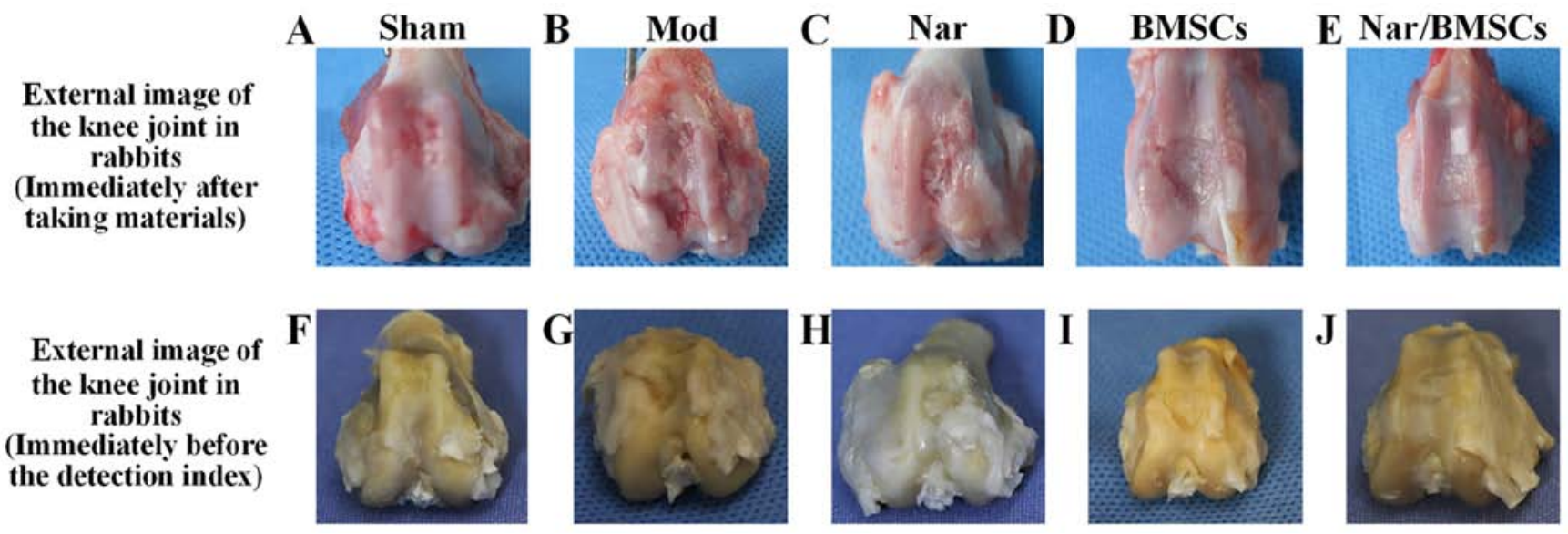

Figure 2. Gross appearance and assessment results of articular cartilage defects in the rabbit models at 12 weeks. General appearance of rabbit knee joints at 12 weeks post-operation, including the (A) Sham group, (B) Mod group, (C) Nar group, (D) BMSCs group and (E) Nar/BMSCs group. General appearance of rabbit knee joints before the histological and immunohistochemical assessment, including the (F) Sham group, (G) Mod group, (H) Nar group, (I) BMSCs group and (J) Nar/BMSCs group. BMSC, bone marrow mesenchymal stem cells; Mod, model; Nar, naringin.

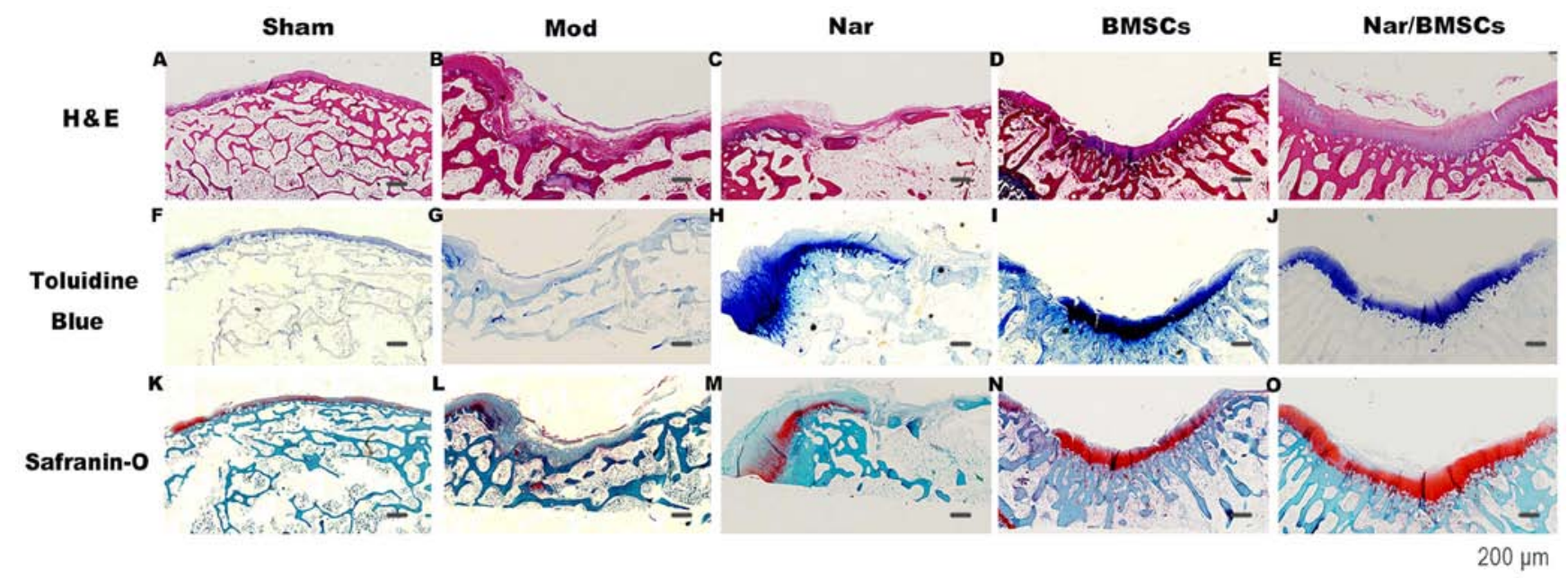

Figure 3. Histological findings of the repair tissue at articular cartilage defect sites ( $\mathrm{n}=8 \mathrm{knees} / \mathrm{group}$ ). Scale bars represent $200 \mu \mathrm{m}$. Representative H\&E staining at 12 weeks in the (A) Sham group, (B) Mod group, (C) Nar group, (D) BMSCs group and (E) Nar/BMSCs group. Toluidine blue staining at 12 weeks in the (F) Sham group, (G) Mod group, (H) Nar group, (I) BMSCs group and (J) Nar/BMSCs group. Safranin-O staining at 12 weeks in the (K) Sham group, (L) Mod group, (M) Nar group, (N) BMSCs group and (O) Nar/BMSCs group. H\&E: Continuous pink on the joint surface illustrates that the cytoplasm of the cartilage is stained pink; Toluidine Blue, continuous dark blue on the joint surface indicates the presence of chondrocytes and cartilage matrix; Safranin $\mathrm{O}$, continuous red on the joint surface indicates the cartilage matrix. BMSC, bone marrow mesenchymal stem cells; H\&E, hematoxylin and eosin; Mod, model; Nar, naringin.

tissue of the Nar/BMSCs group appeared smooth. Differences between regenerative tissues and adjacent normal cartilage were not clear (Fig. 3E). The cartilage and cartilage matrix were evaluated using toluidine blue staining. Cells in the repair tissues of the Mod group were irregularly arranged and full of fibroblast-like cells, without signs of repaired deep tissue or the generation of glycosaminoglycan (GAG; Fig. 3G). The staining of regenerative tissue in the Nar group was clear, but repaired tissues were not completely blended with the adjacent normal cartilage. Although the repair quantity was limited, the repair quality appeared satisfactory (nuclear staining was clear, the cartilage matrix was uniformly distributed; Fig. 3H). The thickness of the stained tissue containing regenerative cells in the BMSCs group was similar to that of the surrounding cartilage. However, the junction of repaired tissue and normal cartilage was still distinguishable in this group (Fig. 3I). The regenerative tissue in the Nar/BMSCs group stained positive and appeared homogeneous, similar to that of the adjacent normal cartilage. The boundary between the normal cartilage and regenerative cartilage was not clear and the cellular morphology and distribution in the new cartilage were almost identical to that of the normal cartilage (Fig. 3J).

Safranin O staining was used to evaluate articular cartilage repair. Joint cartilage was stained in red. The structural repair of the defect site of the BMSCs group was apparent in that Safranin O staining showed a clear structure and the articular surface was almost smooth. However, the cartilage matrix was red and discontinuous and the cartilage repair quality was not complete as the nuclear structure was not clear (Fig. 3N). The articular cartilage regeneration conditions of the groups administered naringin were apparent, the collagen content of the cartilage matrix was clear and there was red staining. The Nar/BMSCs treatment appeared to demonstrate the best 


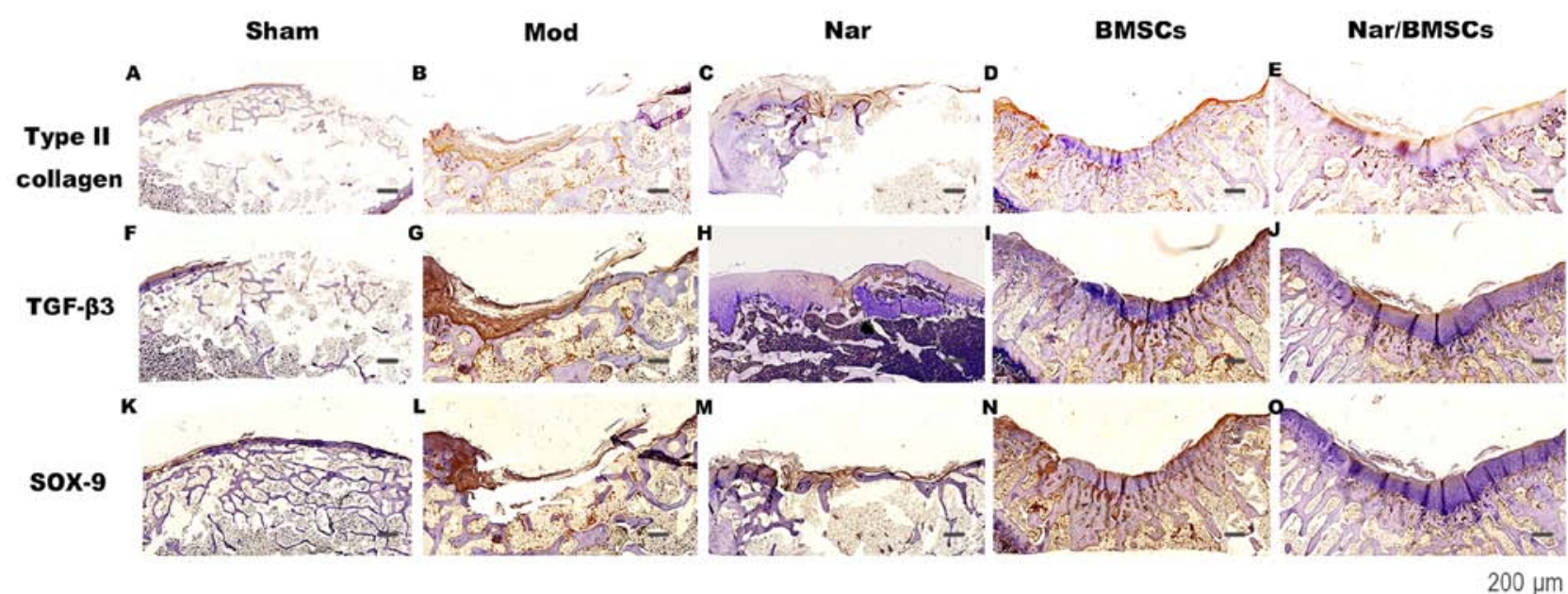

Figure 4. Immunohistological findings of the repair tissue at articular cartilage defect sites ( $\mathrm{n}=8 \mathrm{knees} /$ group). Scale bars represent $600 \mu \mathrm{m}$. Type II collagen immunostaining at 12 weeks in the (A) Sham group, (B) Mod group, (C) Nar group, (D) BMSCs group and (E) Nar/BMSCs group. TGF- $\beta 3$ immunostaining at 12 weeks in the (F) Sham group, (G) Mod group, (H) Nar group, (I) BMSCs group and (J) Nar/BMSCs group. SOX-9 immunostaining at 12 weeks in the (K) Sham group, (L) Mod group, (M) Nar group, (N) BMSCs group and (O) Nar/BMSCs group. Type II collagen, TGF- $\beta 3$ and SOX-9 staining: Brown on the joint surface was positive. BMSC, bone marrow mesenchymal stem cells; Mod, model; Nar, naringin; TGF, transforming growth factor $\beta$.

therapeutic effect. The cartilage matrix was a uniform red and the color of the tissue structure was clear and uniform in the Nar/BMSCs group. The defect site was occupied by cartilage cells, which were highly consistent with the surrounding normal cartilage. Compared with the corresponding position of the defect in Mod group, the appearance of those areas stained in red was similar to that of adjacent normal cartilage (Fig. 3O).

Immunohistochemical staining of the repair tissue on the joint defect site. Type-II collagen immunostaining was used to evaluate the quality of the cartilage repair in the articular cartilage defects. The extracellular matrix of hyaline cartilage cells is mainly composed of type-II collagen and proteoglycan. TGF- $\beta 3$ and SOX- 9 are key factors in the TGF- $\beta$ superfamily signaling pathway. TGF- $\beta 3$ is crucial in cartilage cell proliferation, differentiation and other processes. The expression profile of these markers was evaluated in Fig. 4.

In the Sham group, the expression of type-II collagen was uniform and smooth (Fig. 4A). In the Mod group, there was no significant expression of type-II collagen, with the defect having a sunken surface and uneven tissue (Fig. 4B). In comparison with the Mod group, the BMSCs group presented higher levels of type-II collagen. The Nar and Nar/BMSCs groups, which both received naringin treatment, showed more abundant type-II collagen expression than the group without naringin. The structural repair of joint defect in the Nar group appeared unsatisfactory, some of the joint defects were not repaired, but the content of type-II collagen in the repaired tissue was high, with strong positive staining. Despite a limited number of cells in the area of damage, the cells that were present were mainly regenerative cells of hyaline cartilage (Fig. 4C). Although a clear structural repair of the joint defect was exhibited in the BMSCs group, the type-II collagen content of the repaired tissue was high, with weak positive staining and a limited quantity of regenerative hyaline cartilage (Fig. 4D). The Nar/BMSCs group displayed the best therapeutic effect, the expression of type-II collagen was brownish yellow, and it was superior to other groups in terms of the uniform distribution of lines on the articular surface. Meanwhile, the expression of type-II collagen in Nar/BMSCs group was indicated by strong positive staining and the staining intensity appeared greater than in other groups. The density of chondrocytes and cartilage matrix in the Nar/BMSCs group was similar to that of the normal cartilage. The original defects were filled with novel hyaline cartilage tissue. A possible reason for this is that the implanted BMSCs differentiated into chondrocytes (Fig. 4E).

TGF- $\beta 3$ and SOX-9 are the main indicators of activation of the TGF- $\beta$ superfamily of signaling pathways (32). SOX-9 is regulated and controlled by the TGF- $\beta$ superfamily signaling pathway and serves as the main indicator of downstream signal transmission (33). Areas of positive staining for TGF- 33 in the groups without oral administration of naringin were relatively weak (Fig. 4G and I), while they were stronger in the groups with naringin treatment (Fig. 4J). SOX-9 was uniformly expressed on the surface of the joint in the Sham group (Fig. 4K). Areas of positive staining for SOX-9 in the groups without oral administration of naringin were relatively weak (Fig. 4L). The Nar group showed more SOX-9 expression than the Mod group (Fig. 4M). Although BMSCs group was implanted with bone marrow mesenchymal stem cells, the expression of SOX-9 was lower than in Nar group (Fig. $4 \mathrm{~N})$. While they were stronger in the groups with naringin treatment (Fig. 4O). Notably, the TGF- $\beta 3$ and SOX9 immunohistochemical staining reaction in the Nar/BMSCs group was the most obvious in comparison with that of other groups. The staining was positive in the Nar/BMSCs group, and distributed evenly in the articular surface. The repaired articular defect structure was clear. The regenerative cell matrix of the regenerated hyaline cartilage in the defect repair site was rich in GAG. The arrangement of chondrocytes tended to be regular, and the surface of the cartilage was largely smooth. A satisfactory repair effect can be found from the staining of the above Nar/BMSCs group. (Fig. 4O).

Two professionals conducted quantitative assessment of each sample according to: (i) The International Cartilage 

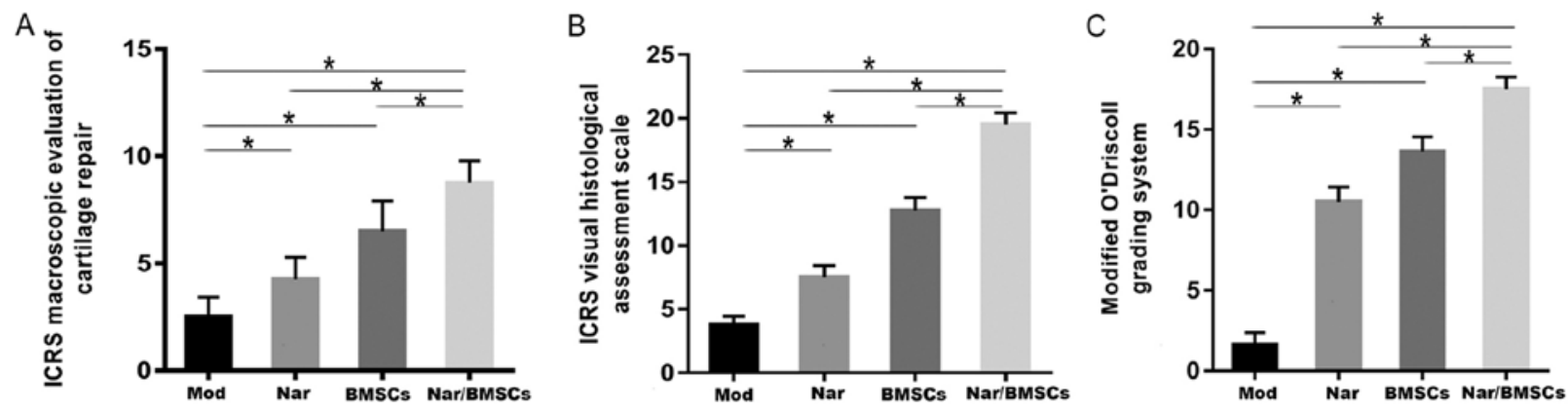

Figure 5. Histological evaluation of the tissue sections. (A) Sections were histologically evaluated based on the ICRS macroscopic evaluation of cartilage repair. (B) Sections were histologically evaluated based on the ICRS Visual Histological Assessment Scale. (C) Sections were histologically evaluated based on modified O'Driscoll grading system. Error bars represent $95 \%$ confidence interval. "P<0.05, BMSC, bone marrow mesenchymal stem cells; ICRS, International Cartilage Repair Society; Mod, model; Nar, naringin.

Repair Society (ICRS) macroscopic evaluation of cartilage repair (Table II),(ii) The ICRS Visual Histological Assessment Scale (Table III) and (iii) the modified O'Driscoll grading system (Table IV). The main parameters assessed were the degree of defect repair, integration to the border zone and macroscopic appearance, surface, matrix and cell distribution, cell population viability, nature of the predominant tissue, structural characteristics and absence of cellular changes due to degeneration. Statistical analysis of the scores of the above items showed that the extent and quality of repair was increased in the Nar group in comparison to the Mod group; in the Nar/BMSCs group in comparison to the BMSCs group; in the BMSCs group in comparison to the Mod group and in the Nar/BMSCs in comparison to the Nar group. In comparison with the other four groups, the Nar/BMSCs group exhibited the best quality of repair, as indicated in Fig. 5.

\section{Discussion}

Knee osteoarthritis is a common clinical disease (34). Articular cartilage injury is the main pathological factor resulting in knee osteoarthritis. Pathological changes in the morphology of the knee cartilage are thought to be the most prominent features during osteoarthritis. Knee cartilage is avascular, aneural and alymphatic, and lacks the ability to self-repair, thus the repair of articular cartilage damage is a challenge for clinicians (35). Cartilage cells serve as the main cellular components of articular cartilage and the articular cartilage surface is mainly formed by hyaline cartilage, which helps to maintain the cartilage cells in an undifferentiated state and to help the cells secrete extracellular matrix (36). The extracellular matrix mainly consists of two types of macromolecules, type-II collagen and proteoglycan.

The structure of normal cartilage when visualized with $\mathrm{H} \& \mathrm{E}$ staining presents a smooth cartilage surface, regular arrangement and almost round chondrocytes. The cartilage matrix under toluidine blue staining appears bluish-purple. The cartilage matrix under safranin $\mathrm{O}$ staining appears dark red. The collagen in cartilage tissue is mainly type II collagen. The brownish yellow part of type II collagen staining can be used as a specific locator of cartilage tissue, which indicates a satisfactory repair of cartilage following injury $(37,38)$.

During the observation of osteoarthritis in the present study, H\&E staining showed unevenly distributed cells, a decrease in the total cell number, apoptosis of the cells and uneven staining. The density of toluidine blue staining was uneven, the staining color was uneven and discoloration was apparent. The phenomenon of light staining or loss of staining appeared in safranin $\mathrm{O}$ staining and the dyeing area decreased. Type-II collagen showed a decreased collagen distribution and superficial nuclear staining.

The BMSCs group displayed typical features of stem cells, which are undifferentiated cells in their original state. They possess a self-replicative ability and a multidirectional differentiation potential, existing in the bone marrow, as well as in other tissues and organs throughout life, and are responsible for the repair and updating of the tissue (39). They can be induced to differentiate into a variety of cells. As the ideal seed cells for cartilage tissue engineering, these cells been studied from numerous perspectives $(40,41)$. The BMSCs in this present study displayed excellent proliferative and differentiation capabilities, as well as a strong viability. This is consistent with the characteristics of BMSCs in previous experiments. BMSCs can be cultured and expanded in order to obtain seed cells, which have been used to treat cartilage defects in numerous animal models $(42,43)$. The use of tissue engineering techniques to study the repair process following cartilage damage is considered a promising approach (2).

Naringin is a component of the TCM treatment, Drynaria fortunei, and is thought to be beneficial for the treatment of osteoarthritis, especially in slowing the occurrence of inflammation (44). Oral administration of naringin has been indicate to have the potential for the treatment of cartilage destruction in osteoarthritis models $(45,46)$. As a multi-functional substance (47), naringin has been suggested to have multiple therapeutic effects, such as the capacity to inhibit the expression of enzymes which are key mediators in the development of osteoarthritis, regulate inflammatory protein secretion and promote cartilage cell infiltration $(48,49)$. These studies highlight the potential of naringin in the treatment of osteoarticular degenerative diseases, which may help to restore the microenvironment of the cartilage defects and improve the state of the tissue. However, studies investigating the function of naringin on signaling pathways in osteoarthritis are rare.

Previous studies have clearly indicated that rabbit BMSCs and naringin solution can be applied to repair rabbit knee articular cartilage defects. The combination of both can help to achieve a better repair (20). However, the specific mechanisms 
of action remain unknown; therefore, further investigation into the repair mechanisms would be of great clinical significance.

Biological processes such as the formation, metabolism and apoptosis of articular cartilage are regulated by multiple signaling pathways, among which, the TGF- $\beta$ superfamily signaling pathway is prominent in maintenance of the articular cartilage, through regulating mesenchymal cell migration, aggregation and differentiation into cartilage cells; and through regulating the formation of cartilage matrix (50). There are three types of mammalian TGF- $\beta$, namely $\beta 1, \beta 2$ and $\beta 3$ (51). The TGF- $\beta$ superfamily signaling pathway is important in the development of the growth plate and the formation of permanent articular cartilage. Previous studies have indicated that TGF- $\beta$ plays an important regulatory role in the maintenance of the normal cartilage cell phenotype, as well as in the promotion of proliferating cells to differentiate into mature cartilage cells (52-54). Similarly, the TGF- $\beta$ signaling pathway helps to maintain the cartilage cell phenotype and the stability of the cartilage tissue environment (55). Not only in mature cartilage, but also in the process of cartilage formation, blocking the TGF- $\beta$ superfamily signaling pathway has been shown to have harmful effects (56). TGF- $\beta 3$ has a stronger ability to induce MSCs to differentiate into cartilage than that of TGF- $\beta 1(57,58)$. Therefore, TGF- $\beta 3$ was selected as one of the main evaluation indexes of pathway activation in the present study.

SOX-9 can be regulated by the TGF superfamily signalling pathway. TGF- $\beta$ can promote the proliferation and differentiation of cartilage cells by maintaining SOX-9 expression levels (59). SOX-9 is thought to be one of the effective transcription factors that are regulated by type-II collagen and is deemed a modular switch of cartilage differentiation (60). SOX9 is a key transcription factor in development and is active during chondrogenic differentiation, it directly binds to enhancers in the chondrocyte genome by homodimerizing on pairs of 7-bp SOX-domain DNA recognition sites (61). Moreover, it is a key transcription factor of signaling pathways which induce cartilage differentiation of BMSCs (62). During the cartilage differentiation process, SOX-9 can be combined with the enhancer element of the type-II collagen gene to increase the expression of type-II collagen (63) and to promote the differentiation of MSCs into chondrocytes (64). The absence of SOX-9 results in the failure of MSCs to differentiate into cartilage cells, leading to damage to the cartilage primordium (65). Sufficient SOX-9 expression levels can not only facilitate the enrichment of MSCs but can also help to inhibit the conversion of proliferated cartilage cells into a mast cell phenotype. The maintenance of SOX-9 expression levels highly depends on the continuous signal stimulation of the TGF- $\beta$ superfamily signaling pathway. In summary, Sox-9 has a positive regulating function on the differentiation and maturation of the cartilage. Therefore, SOX-9 was selected as one of the downstream evaluation indexes of pathway activation in the present study $(32,66)$.

The BMSCs group in the present study was found to repair the articular cartilage defect of the rabbit knee and the therapeutic effect on articulation structure repair was deemed satisfactory; however, the curative effect on the cartilage quality repair was poor, which was related to the inactivation of the TGF- $\beta$ superfamily signaling pathway. Compared with that of the Mod group, the BMSC group prominently improved the degree of defect repair and integration to the border zone, the defective joint structure was restored to a certain degree. In the BMSCs group, BMSCs implanted at articular cartilage defects may repair the structures of the joint. However, restoring the structures did not appear to be sufficient and the quality of the repair was important. The quality of repair was significantly different between the regenerated tissue of the BMSCs group and the normal cartilage. The content of hyaline cartilage in the regenerated tissue was lower than that in the repaired tissue, which indicated that the BMSCs were not satisfactory in terms of the repair quality of the articular cartilage defect.

The expression levels of TGF- $\beta 3$ and SOX-9 in the Mod and BMSCs groups were relatively low, which indicated that the BMSCs did not activate the TGF- $\beta$ superfamily signaling pathway during the repair of the articular cartilage defects in rabbit knees. Naringin, the active ingredient of the TCM treatment Drynaria fortune (67), was effective at improving the quality of the repair of the articular cartilage defects in the rabbit knees, which may be related to the activation of the TGF- $\beta$ superfamily signaling pathway. However, its effect on repairing the defected joint structure was not deemed satisfactory, which may be attributed to the lack of adequate repair cells.

Although the Nar group's contour repair for joint defects was stronger than that of the Mod group, the joint defects did not demonstrate a satisfactory level of repair to the structure. This could be attributed to an insufficient number of repairing seed cells in the articular cartilage defect and could also be related to a poor intrinsic healing potential $(68,69)$. Drug treatment alone appears to be unable to significantly promote the recovery of the joint defect structure. Toluidine blue and safranin $\mathrm{O}$ staining revealed that, compared with that of the Mod group, the Nar group had significantly improved cartilage quality in the newly repaired tissue at the defect site. According to the results of type-II collagen staining, the newly repaired tissue was composed of predominantly hyaline cartilage. These findings demonstrated that naringin helped to promote an improved quality of cartilage regeneration and repair in articular cartilage defects of the rabbit knees.

The expression levels of TGF- $\beta 3$ and SOX-9 were higher in the Nar group compared with those in the Mod group, which indicated that naringin stimulated the secretion of these two specific factors. TGF- $\beta 3$ can act as the chemotactic molecule that induces chondrogenesis (70). A study into Sox9 post-translational regulation found that the Sox 9 protein levels were closely related to the formation of normal cartilage (62). Moreover, SOX-9 can be used as a marker of cartilage formation in degenerated cartilage cells. These data demonstrate that naringin, the effective ingredient of the TCM treatment Drynaria fortunei, may be effective at promoting repair of the articular cartilage defects in rabbit knees through activation of the TGF- $\beta$ superfamily signaling pathway.

BMSCs together with naringin, can promote the repair of articular cartilage defects in rabbit knees. The repair of the joint structure defects and the quality of the repair of the articular cartilage were both deemed satisfactory and were found to be enhanced compared to that of the individual application of BMSCs or naringin. This may be associated with the simultaneous activation of the TGF- $\beta$ superfamily signaling pathway in the presence of sufficient seed cells. 
Both the BMSCs and the Nar/BMSCs groups and exhibited satisfactory effects on the structural repair of the joint defects. Regarding the degree of the defect repair and the association with the surrounding tissues, the Nar/BMSCs group presented greater improvements than the BMSCs group. The Nar/BMSCs group displayed better repair effects in articular cartilage regeneration and cartilage matrix repair as a result of the treatment with naringin. Regarding type-II collagen, the Nar/BMSCs group appeared identical to the surrounding normal articular cartilage tissue and the repaired tissue consisted of a considerable quantity of type-II collagen. The repaired tissue maintained a fairly smooth surface profile and its hyaline cartilage content was higher than that of the BMSCs group. This indicated that naringin may increase the directional differentiation of BMSCs into cartilage tissue in the articular cartilage defect. Furthermore, naringin improved the quality of cartilage regeneration and repair in articular cartilage defects of the rabbit knees.

The expression levels of TGF- $\beta 3$ and SOX-9 were significantly higher in the Nar/BMSCs group compared to that in the BMSCs group. Particularly, compared to all other groups in the present study, the expression levels of TGF- $\beta 3$ and SOX-9 were highest in the Nar/BMSCs group. This is consistent with the conclusion that Sox-9 has the potential to restore degenerated cartilage structure and functions (71).

The BMSCs implanted in the articular cartilage defects in the rabbit knees appeared to provide sufficient seed cells for repair to occur. Oral administration of naringin exerted a continuous regulatory function on the TGF- $\beta$ superfamily signaling pathway, which promoted the directional differentiation of BMSCs into cartilage tissue at articular cartilage defects in rabbit knees. Notably, the majority of the differentiated cartilage cells expressed a hyaline cartilage phenotype.

The present study demonstrated that BMSCs had a satisfactory effect on the repair of the joint structure of articular cartilage defects in rabbit knees. Naringin helped to improve the quality of cartilage repair in articular cartilage defects in rabbit knees. BMSCs combined with naringin, appeared to further improve the repair of articular cartilage defects in rabbit knees, thus delivering satisfactory results for joint structure and cartilage quality. It appears that the repair mechanism of action of naringin on articular cartilage defects may be through the continuous regulation of the TGF- $\beta$ superfamily signaling pathway and that combined application with BMSCs may produce better results than the use of naringin alone. These findings may be attributed to the directional differentiation of BMSCs into cartilage cells after regulation of the TGF- $\beta$ superfamily signaling pathway.

The present study is a preliminary study focused on articular cartilage defect repair of rabbit knees using a combination of naringin and BMSCs. Future research will investigate the effects of different concentrations of naringin on articular cartilage defect repair in rabbit knees, the target tissue of naringin (such as chondrocytes or cartilage matrix) and the direct effects of naringin on cartilage cells. Furthermore, the direct functional mechanism of action of naringin on the TGF- $\beta$ superfamily signaling pathway and the mechanism of naringin's influence on BMSCs should be further investigated.

In conclusion, naringin, an effective ingredient of the TCM Drynaria fortunei, and BMSCs were able to stimulate the repair of articular cartilage defects in rabbit knees. The combined application of these treatments on joint structural repair and articular cartilage repair quality delivered improved results, which may be closely associated with sustained positive regulation of the TGF- $\beta$ superfamily signaling pathway and with the directional differentiation of BMSCs into cartilage cells.

\section{Acknowledgements}

The authors would like to thank Professor Mingjing Zhao (Key Laboratory of Chinese Internal Medicine of Ministry of Education and Beijing, Dongzhimen Hospital, Beijing University of Chinese Medicine, Beijing, China) for technical assistance with this study.

\section{Funding}

This study was supported by the Fundamental Research Funds for the Central Universities (grant nos. 2015-JYB-JSMS058, 2 018-JYBZZ-JS091).

\section{Availability of data and materials}

The datasets used and/or analyzed during the current study are available from the corresponding author on reasonable request.

\section{Authors' contributions}

PL conceived and designed the study. CY, JC, HL, JY and FW performed the animal surgeries. YL, CY and PL performed the histological and immunohistochemical staining. CY, JC, HL, JY, ZY and YQ acquired the data. CY, JC, ZY and PL analyzed and interpreted data. CY and JC drafted the manuscript. PL and ZY critically revised the manuscript for important intellectual content. All authors read and approved the final manuscript.

\section{Ethics approval and consent to participate}

The animal experiments were approved by the Science and Technology Department of Beijing University of Chinese Medicine (Beijing, China) and the Animal Ethics Review Committee of the Institute of Basic Theories of Chinese Medicine, Chinese Academy of Chinese Medical Sciences (Beijing, China) approval no. 201706058.

\section{Patient consent for publication}

Not applicable.

\section{Competing interests}

The authors declare that they have no competing interests.

\section{References}

1. Zhu Y, Yuan M, Meng HY, Wang AY, Guo QY, Wang Y and Peng J: Basic science and clinical application of platelet-rich plasma for cartilage defects and osteoarthritis: A review. Osteoarthritis Cartilage 21: 1627-1637, 2013. 
2. Browne JE and Branch TP: Surgical alternatives for the treatment of articular cartilage lesions. J Am Acad Orthop Sur 8: 180-189, 2000.

3. Katagiri H, Mendes LF and Luyten FP: Definition of a critical size osteochondral knee defect and its negative effect on the surrounding articular cartilage in the rat. Osteoarthritis Cartilage 25: 1531-1540, 2017.

4. Wang AT, Feng Y, Jia HH, Zhao M and Yu H: Application of mesenchymal stem cell therapy for the treatment of osteoarthritis of the knee: A concise review. World J Stem Cells 11: 14-27, 2019

5. Ebihara G, Sato M, Yamato M, Mitani G, Kutsuna T, Nagai T, Ito S, Ukai T, Kobayashi M, Kokubo M, et al: Cartilage repair in transplanted scaffold-free chondrocyte sheets using a minipig model. Biomaterials 33: 3846-3851, 2012.

6. Armiento AR, Alini M and Stoddart MJ: Articular fibrocartilage-Why does hyaline cartilage fail to repair? Adv Drug Deliv Rev 146: 289-305, 2019.

7. Bae DK, Yoon KH and Sang JS: Cartilage healing after microfracture in osteoarthritic knees. Arthroscopy 22: 367-374, 2006.

8. Wang Y, Yuan M, Guo QY, Lu SB and Peng J: Mesenchymal stem cells for treating articular cartilage defects and osteoarthritis. Cell Transplant 24: 1661-1678, 2015.

9. Che CT, Wong MS and Lam CW: Natural products from chinese medicines with potential benefits to bone health. Molecules 21: 239, 2016.

10. An J, Yang H, Zhang Q, Liu C, Zhao J, Zhang L and Chen B: Natural products for treatment of osteoporosis: The effects and mechanisms on promoting osteoblast-mediated bone formation. Life Sci 147: 46-58, 2016.

11. Su YX, Yan H, Chen BJ, Zahn Q, Wang YR, Lu ML, Wang WT, He $Z$ and Sheng L: Effect of naringin of Drynaria Rhizome, a Chinese medical component of Zhuanggu Jianxi recipe containing serum on Caveolin-p38MAPK signal pathway in IL-1 $\beta$ induced rabbit degenerated chondrocytes. Zhongguo Zhong Xi Yi Jie He Za Zhi 34: 1492-1498, 2014 (In Chinese).

12. Lo PC, Lin FC, Tsai YC and Lin SK: Traditional Chinese medicine therapy reduces the risk of total knee replacement in patients with knee osteoarthritis. Medicine (Baltimore) 98: e15964, 2019.

13. Teixeira J, Santos MJ, Matos LC and Machado JP: Evaluation of the effectiveness of acupuncture in the treatment of knee osteoarthritis: A case study. Medicines (Basel) 5: 18, 2018.

14. An J, Hao D, Zhang Q, Chen B, Zhang R, Wang Y and Yang H: Natural products for treatment of bone erosive diseases: The effects and mechanisms on inhibiting osteoclastogenesis and bone resorption. Int Immunopharmacol 36: 118-131, 2016.

15. Chen R, Qi QL, Wang MT and Li QY: Therapeutic potential of Naringin: An overview. Pharm Biol 54: 3203-3210, 2016

16. Farrell MJ, Shin JI, Smith LJ and Mauck RL: Functional consequences of glucose and oxygen deprivation on engineered mesenchymal stem cell-based cartilage constructs. Osteoarthritis Cartilage 23: 134-142, 2015.

17. Wolf D and Wolf AM: Mesenchymal stem cells as cellular immunosuppressants. Lancet 371: 1553-1554, 2008.

18. Santo VE, Gomes ME, Mano JF and Reis RL: Controlled release strategies for bone, cartilage, and osteochondral engineering-Part II: Challenges on the evolution from single to multiple bioactive factor delivery. Tissue Eng Part B Rev 19: 327-352, 2013.

19. Cha BH, Kim JH, Kang SW, Do HJ, Jang JW, Choi YR, Park H, Kim BS and Lee SH: Cartilage tissue formation from dedifferentiated chondrocytes by codelivery of BMP-2 and SOX-9 genes encoding bicistronic vector. Cell Transplant 22 $1519-1528,2013$

20. Paul R, Haydon RC, Cheng H, Ishikawa A, Nenadovich N, Jiang W, Zhou L, Breyer B, Feng T, Gupta P, et al: Potential use of Sox9 gene therapy for intervertebral degenerative disc disease. Spine (Phila Pa 1976) 28: 755-763, 2003.

21. Tew SR, Pothacharoen P, Katopodi T and Hardingham TE: SOX9 transduction increases chondroitin sulfate synthesis in cultured human articular chondrocytes without altering glycosyltransferase and sulfotransferase transcription. Biochem J 414: 231-236, 2008

22. Li PY, Li CG, Ye C, Qu Y, Chen J, Zhu GQ and Zhao H: Experimental study on Naringin decoction combined with bone marrow mesenchymal stromal stem cells in repair of articular cartilage defects in rabbits. J Liaoning Univ Tradit Chinese Med 16: 34-38, 2014.

23. Li CG, Qu Y, Ye C, Chen J, Wang FX, Li PY, Li SH, Ren JP and Qi J: Histology research on repairing of rabbit articular cartilage defects with Naringin and tissue engineering cartilage. Chin J Tissue Eng Res 18: 3165-3171, 2014.
24. Cai YT, Liu TS, Fang F, Xiong CL and Shen SL: Comparisons of mouse mesenchymal stem cells in primary adherent culture of compact bone fragments and whole bone marrow. Stem Cells Int 2015: 708906, 2015.

25. Parodi AL: Ethical issue in animal experimentation. Bull Acad Natl Med 193: 1737-1745, 2009 (In French)

26. Wei W, Wu XM and Li YJ: Pharmacological Experimental Methodology. 4th edition. People's Medical Publishing House, pp69-73, 2010.

27. Gao XM: Science of Chinese Materia Medica. 2nd edition. China Press of Traditional Chinese Medicine, pp335-336, 2007.

28. Smith GD, Taylor J, Almqvist KF, Erggelet C, Knutsen G, Garcia Portabella M, Smith T and Richardson JB: Arthroscopic assessment of cartilage repair: A validation study of 2 scoring systems. Arthroscopy 21: 1462-1467, 2005.

29. van den Borne MP, Raijmakers NJ, Vanlauwe J, Victor J, de Jong SN, Bellemans J and Saris DB; International Cartilage Repair Society: International Cartilage Repair Society (ICRS) and Oswestry macroscopic cartilage evaluation scores validated for use in Autologous Chondrocyte Implantation (ACI) and microfracture. Osteoarthritis Cartilage 15: 1397-1402, 2007.

30. Mainil-Varlet P, Aigner T, Brittberg M, Bullough P, Hollander A, Hunziker E, Kandel R, Nehrer S, Pritzker K, Roberts S, et al: Histological assessment of cartilage repair: A report by the histology endpoint committee of the international car-tilage repair society (ICRS). J Bone Joint Surg Am 85A (Suppl 2): S45-S57, 2003.

31. O'Driscoll SW, Keeley FW and Salter RB: The chondrogenic potential of free autogenous periostal grafts for biological resurfacing of major full-thickness defects in joint surfaces under the influence of continuous passive motion. An experimental investigation in the rabbit. J Bone Joint Surg Am 68: 1017-1035, 1986.

32. Coricor $\mathrm{G}$ and Serra R: TGF- $\beta$ regulates phosphorylation and stabilization of Sox9 protein in chondrocytes through p38 and Smad dependent mechanisms. Sci Rep 6: 38616, 2016.

33. Ni Q, Lu K, Li J, Tan Y, Qin J, Magdalou J, Chen L and Wang H: Role of TGF $\beta$ signaling in maternal ethanol-induced fetal articular cartilage dysplasia and adult onset of osteoarthritis in male rats. Toxicol Sci 164: 179-190, 2018

34. Johnson K, Zhu S, Tremblay MS, Payette JN, Wang J, Bouchez LC, Meeusen S, Althage A, Cho CY, Wu X and Schultz PG: A stem cell-based approach to cartilage repair. Science 336: 717-721, 2012.

35. Bekkers JE, Creemers LB, Tsuchida AI, van Rijen MH, Custers RJ, Dhert WJ and Saris DB: One-stage focal cartilage defect treatment with bone marrow mononuclear cells and chondrocytes leads to better macroscopic cartilage regeneration compared to microfracture in goats. Osteoarthritis Cartilage 21: 950-956, 2013.

36. Caron MM, Emans PJ, Coolsen MM, Voss L, Surtel DA, Cremers A, van Rhijn LW and Welting TJ: Redifferentiation of dedifferentiated human articular chondrocytes: Comparison of 2D and 3D cultures. Osteoarthritis Cartilage 20: 1170-1178, 2012.

37. Yu F, Zeng H, Yu HY, Lei M, Yuan H and Xiao DM: Comparison of different special staining techniques of chondrocytes and their application values. Chinese J Comp Med 8: 58-61, 2015.

38. Yu F, Lei M, Zeng H, Cao JF and Xiao DM: Different special staining methods on cartilage tissue of osteoarthritis morphology: A comparison study. Orthop J China 19: 79-85, 2015.

39. Chamberlain G, Fox J, Ashton B and Middleton J: Concise review: Mesenchymal stem cells: Their phenotype, differentiation capacity, immunological features, and potential for homing. Stem Cells 25: 2739-2749, 2010.

40. Kock L, Donkelaar CCV and Ito K: Tissue engineering of functional articular cartilage: The current status. Cell Tissue Res 347: 613-627, 2012.

41. Caplan AI: Review: Mesenchymal stem cells: Cell-based reconstructive therapy in orthopedics. Tissue Eng 11: 1198-1211, 2005.

42. Anraku Y, Mizuta H, Sei A, Kudo S, Nakamura E, Senba K, Takagi $\mathrm{K}$ and Hiraki Y: The chondrogenic repair response of undifferentiated mesenchymal cells in rat full-thickness articular cartilage defects. Osteoarthritis Cartilage 16: 961-964, 2008.

43. Kurth TB, Dell'Accio F, Crouch V, Augello A, Sharpe PT and De Bari C: Functional mesenchymal stem cell niches in adult mouse knee joint synovium in vivo. Arthritis Rheumatol 63: 1289-1300, 2014

44. Manna K, Das U, Das D, Kesh SB, Khan A, Chakraborty A and Dey S: Naringin inhibits gamma radiation-induced oxidative DNA damage and inflammation, by modulating p 53 and NF- $\kappa B$ signaling pathways in murine splenocytes. Free Radical Res 49: 422-439, 2015 . 
45. Zhao Y, Zhong L, Wang W, Zhang H, Chen J, Su, P, Liu L and Li W: Naringin protects against cartilage destruction in osteoarthritis through repression of NF- $\mathrm{KB}$ signaling pathway. Inflammation 39: 385-392, 2016.

46. Xu Q, Zhang ZF and Sun WX: Effect of naringin on monosodium iodoacetate-induced osteoarthritis pain in rats. Med Sci Monitor 23: 3746-3751, 2017.

47. Cao X, Lin W, Liang C, Zhang D, Yang F, Zhang Y, Zhang X, Feng J and Chen C: Naringin rescued the TNF- $\alpha$-induced inhibition of osteogenesis of bone marrow-derived mesenchymal stem cells by depressing the activation of $\mathrm{NF}-\mathrm{KB}$ signaling pathway. Immunol Res 62: 357-367, 2015.

48. Kawaguchi K, Maruyama H, Hasunuma R and Kumazawa Y: Suppression of inflammatory responses after onset of collagen-induced arthritis in mice by oral administration of the Citrus flavanone naringin. Immunopharm Immunot 33: 723-729, 2011.

49. Ahmad SF, Zoheir KM, Abdelhamied HE, Ashour AE, Bakheet SA, Attia SM and Abd-Allah AR: Amelioration of autoimmune arthritis by Naringin through modulation of T regulatory cells and Th1/Th2 cytokines. Cell Immunol 287: 112-120, 2014.

50. Ashraf S, Cha BH, Kim JS, Ahn J, Han I, Park H and Lee SH: Regulation of senescence associated signaling mechanisms in chondrocytes for cartilage tissue regeneration. Osteoarthritis Cartilage 24: 196-205, 2016.

51. Feng XH and Derynck R: Specificity and versatility in tgf-beta signaling through Smads. Annu Rev Cell Dev Bi 21: 659-693, 2005.

52. Dong R, Ying J, Xu TT, Hu SF, Zhang P, Xia CJ, Fang L, Jin HT and Wang PG. Bushenhuoxue formula facilitates articular cartilage repair and attenuates matrix degradation by activation of TGF- $\beta$ signaling pathway. Evid Based Complement Alternat Med 2018: 2734581, 2018.

53. Patil AS, Sable RB and Kothari RM: An update on transforming growth factor- $\beta$ (TGF- $\beta$ ): Sources, types, functions and clinical applicability for cartilage/bone healing. J Cell Physiol 226: 3094-3103, 2011.

54. Grimaud E, Heymann D and Rédini F: Recent advances in TGF-beta effects on chondrocyte metabolism. Potential therapeutic roles of TGF-beta in cartilage disorders. Cytokine Growth Factor Rev 13: 241-257, 2002.

55. Blaney Davidson EN, van der Kraan PM and van den Berg WB TGF-beta and osteoarthritis. Osteoarthritis Cartilage 15: 597-604, 2007

56. Li YP, Wei XC, Zhou JM and Wei L: The age-related changes in cartilage and osteoarthritis. Biomed Res Int 2013: 916530, 2013.

57. Mackay AM, Beck SC, Murphy JM, Barry FP, Chichester CO and Pittenger MF: Chondrogenic differentiation of cultured human mesenchymal stem cells from marrow. Tissue Eng 4: 415-428, 1998

58. Lu CH, Lin KJ, Chiu HY, Chen CY, Yen TC, Hwang SM, Chang $\mathrm{YH}$ and $\mathrm{Hu} \mathrm{YC}$ : Improved chondrogenesis and engineered cartilage formation from TGF- $\beta 3$-expressing adipose-derived stem cells cultured in the rotating-shaft bioreactor. Tissue Eng Part A 18: 2114-2124, 2012.
59. Furumatsu T, Tsuda M, Taniguchi N, Tajima $Y$ and Asahara $H$ : Smad3 induces chondrogenesis through the activation of SOX9 via CREB-binding protein/p300 recruitment. J Biol Chem 280: 8343-8350, 2005.

60. Sha'ban M, Cassim SO, Yahya NM, Saim A and Idrus R: Sox-9 Transient transfection enhances chondrogenic expression of osteoarthritic human articular chondrocytes in vitro: Preliminary analysis. J Tissue Eng Regen 8: 32-41, 2011.

61. Liu CF and Lefebvre V: The transcription factors SOX9 and SOX5/SOX6 cooperate genome-wide through super-enhancers to drive chondrogenesis. Nucleic Acids Res 43: 8183-8203, 2015.

62. Kawakami Y, Rodriguez-León J and Izpisúa Belmonte JC: The role of TGFbetas and Sox9 during limb chondrogenesis. Curr Opin Cell Biol 18: 723-729, 2006.

63. Lefebvr V, Huang W, Harley VR, Goodfellow PN and De CB: SOX9 is a potent activator of the chondrocyte-specific enhancer of the pro alpha1(II) collagen gene. Mol Cell Biol 17: 2336-2346, 1997.

64. Wang Z, Li K, Sun H, Wang J, Fu Z and Liu M: Icariin promotes stable chondrogenic differentiation of bone marrow mesenchymal stem cells in self-assembling peptide nanofiber hydrogel scaffolds. Mol Med Rep 17: 8237-8243, 2018.

65. Yoon BS, Ovchinnikov DA, Yoshii I, Mishina Y, Behringer RR and Lyons KM: Bmprla and Bmprlb have overlapping functions and are essential for chondrogenesis in vivo. Proc Natl Acad Sci USA 102: 5062-5067, 2005.

66. Lefebvre V and Dvir-Ginzberg M: SOX9 and the many facets of its regulation in the chondrocyte lineage. Connect Tissue Res 58: 2-14, 2017.

67. Lee YE, Liu HC, Lin YL, Liu SH, Yang RS and Chen RM: Drynaria fortune J. Sm. improves the bone mass of ovariectomized rats through osteocalcin-involved endochondral ossification. J Ethnopharmacol 158: 94-101, 2014.

68. Hunziker EB, Lippuner K, Keel MJ and Shintani N: An educational review of cartilage repair: Precepts and practice - myths and misconceptions - progress and prospects. Osteoarthritis Cartilage 23: 334-350, 2015.

69. Huey DJ, Hu JC and Athanasiou KA: Unlike bone, cartilage regeneration remains elusive. Science 338: 917-921, 2012.

70. Hara ES, Ono M, Hai PT, Sonoyama W, Kubota S, Takigawa M, Matsumoto T, Young MF, Olsen BR and Kuboki T: Fluocinolone acetonide is a potent synergistic factor of TGF- $\beta 3$-associated chondrogenesis of bone marrow-derived mesenchymal stem cells for articular surface regeneration. J Bone Miner Res 30: 1585-1596, 2015.

71. Tew SR, Li Y, Pothacharoen P, Tweats LM, Hawkins RE and Hardingham TE: Retroviral transduction with SOX9 enhances re-expression of the chondrocyte phenotype in passaged osteoarthritic human articular chondrocytes. Osteoarthritis Cartilage 13: 80-89, 2005.

This work is licensed under a Creative Commons Attribution-NonCommercial-NoDerivatives 4.0 International (CC BY-NC-ND 4.0) License. 